\title{
Degradation of Low Density Polyethylene Due To Successive Exposure to Acid Rain and UV Radiation
}

\author{
M.M. El-Tonsy \\ Physics Dept \\ Faculty of Science \\ Mansoura University \\ Al-Mansoura, Egypt
}

\author{
S. A. A. AlSaati \\ University of Babylon \\ Iraq
}

\author{
A. H. Oraby \\ Physics Dept \\ Faculty of Science Al- \\ Mansoura University \\ 35516 Egypt
}

\begin{abstract}
Utilization of polymer products for outdoor applications is continuously increasing. So the stability of polymers against environmental degradation became top of interests for many researchers. The effect of environmental elements on the polymers stability has been studied, but individually. A solution against an environmental element may conflict with a solution against other element. Therefore current study aimed to clarify a sort of these conflicts, by successive exposure of low density polyethylene (LDPE) films to acid rains and ultra violet (UV) radiation for different times. The used LDPE films are selected from the commercial grads which are used for plants greenhouses, in order to use samples fully protected against environmental elements. It is found that acid rains etch PE films, causing removal for some of the UV stabilizer additives, and hence UV radiation could attack PE films seriously causing remarked oxidative degradation. This study includes wide comparisons between effects of acid rain only, UV irradiation only, acid rain followed by UV irradiation and UV irradiation followed by acid rain exposure. Variations in the chemical composition, morphological structures, thermal and mechanical properties are detected by the IR- spectroscopy, X-ray diffraction, differential thermal analysis (DTA) and tensile tests. A new view for the differentiation between degradations caused by acid rains and UV radiation is discussed. Lot of experimental data are given in many coloured graphs and tables..
\end{abstract}

\section{GENERAL VIEW AND OBJECTIVE}

When sulphur dioxide and nitrogen oxides are emitted into the atmosphere, they come into contact with water where they are chemically converted to acidic compounds of sulphates and nitrates. These strong acids are deposited onto the earth's surface as rain, snow and fog and through dry deposition; the name "acid rain" is commonly used for describing this painful mechanism. Sulphur is released when fossil fuels are burned, mainly for electricity production and industrial processes. Oxides of nitrogen are released during burning of all fossil fuels too, including gasoline and diesel fuel, where the nitrogen in the fuel and atmosphere reacts with oxygen. Acid rain contaminates drinking water, damages buildings, by corroding cement, accelerates plastics degradation, and causes metals to rust.

Of the solar wavelengths, the UV-B component is particularly efficient in bringing about photo-damage in synthetic and naturally occurring materials. This is particularly true of plastics, rubber and wood used in the building and agricultural industries. Solar radiation spectrum ranges over 290-300 nm and it consists of just less than $10 \%$ of UV radiation but it has sufficient energies to dissociate $\mathrm{C}-\mathrm{C}$ or $\mathrm{C}-\mathrm{H}$ bonds in polymer. The free radicals produced in this way may then react freely with the atmospheric oxygen and contribute to further degradation of the polymer, which is called photo-oxidation.

The outdoor service life of common plastic materials is limited by their withstanding solar ultraviolet radiation and other environmental conditions like the acid rain. Many researching efforts have been carried out for evaluating the serious effects of solar UV and acid rains individually, although both environmental elements are acting either simultaneously or successively. As a result, several protection solutions are proposed against each individual element regardless the efficiency of this protection solution in presence of the other element. In the current work, a polymer sample is exposed to acid rain and UV radiation in sequenced manner and the net effects were recorded and analysed. This work aims to present more information to increase the efficiency of polymers protection against all environmental conditions on time. This work tries to show how the UV radiation may restrict the polymer protection against the acid rains attack, and vice versa.

\section{INTRODUCTION}

Plastics have been employed in agriculture because of their unique properties in comparison with glass, like unbreakability, low price, transparency, flexibility and light weight. Plastics (low density polyethylene, LDPE or polypropylene PP) are now widely used for the construction of plants green houses. In this case plastic films are exposed to the most aggressive environmental conditions. The greenhouse plastic films are attacked by the solar UV radiation along the day time. Many days or weeks around the year, acidic rains attack these films too. Thus the two environmental elements acid rain and UV radiation are attacking the greenhouses plastic films either simultaneously or successively. The successive exposure to these environmental elements is the most dominant case. 
The degradation processes of the various types of polymer have specific mechanisms and depend on both the main macromolecular chain nature, structure and on the chemical nature of the branched groups. It is well known, for the oxidation of polyolefin's or rubber (natural and some synthetic types), that hydro-peroxides and the peroxy radicals are intermediates in a long kinetic chain mechanism. In the case of the halogenated polymers the de-hydro-halogenations is the first reaction step followed by the thermal oxidation, while for the polyamides or cellulose the oxidation occurs by hydroperoxides within a short chain mechanism [1-7].

Two main groups of factors are causing the most polymers degradations, which are:

1 - Chemical factors: oxygen, chemicals, ozone, polymerization catalysts, etc.

2 - Physical factors: light, ionizing radiation, heat, mechanical processing, etc.

The degradation process starts by releasing a free radical either by energetic photon or by breaking a chemical bond thermally or chemically. The is the so called initiating the process which is repeated several times as long as the initiator is active, as shown in figure 1 .<smiles>CCCCCCCC</smiles>

Figure (1): The hydrogen is separated from the polymer chain leaving hydrogen radical (H.) and polymer chain radical (C.).

It is also possible for the $\mathrm{C}-\mathrm{C}$ bonds to be broken, figure 2 :<smiles>CCCCCCC</smiles>

Figure (2): Breaking the C-C bond.

but due to the energy required it is less likely to occur [8]. If the photo-irradiation takes place and oxygen is available then photo-oxidation reactions are able to continue as shown down:

The highly reactive polymer radical $\left(\mathrm{C}^{*}\right)$ is able to react with oxygen resulting in the formation of a peroxy polymer radical $\left(\mathrm{COO}^{*}\right)$. Two of the hydrogen radicals $\left(\mathrm{H}^{*}\right)$ are able to react together resulting in the release of hydrogen gas $(\mathrm{H} 2)$. The rate of formation of these peroxy radicals is controlled by the propagation of oxygen into the polymer. The newly formed peroxy radical (COO*) is able to extract hydrogen from the polymer chain to form hydro-peroxide and another polymer radical $\left(\mathrm{C}^{*}\right)$, as shown down:

These hydro-peroxides are unstable and so will decompose to form further radicals from the continued UV radiation [9]. Another product commonly associated with oxidation is the production of carbonyl groups $(\mathrm{C}=\mathrm{O})$, the formation of which can result in a range of new molecules. The formation of carbonyl groups is also thought to play a key role in the chain scission of the polymer back bone [10]. The formation of a simple ketone group which is measurable by IR spectroscopy with absorption at $1722 \mathrm{~cm}-1$ is common. Another common carbonyl group seen in photo-oxidised LDPE is aldehyde, measurable by IR spectroscopy with an increased absorption at $1730 \mathrm{~cm}-1$.The formation is thought to typically result in the scission of the polymer chain.

Polyethylene (PE) resins in their natural state are inherently unstable and degrade when exposed to oxygen (air oxygen). The degradation is similar to the rusting (or oxidation) of untreated iron in that the polymers change colour to yellowbrown and begin to flake away until the material becomes useless. When PE degrades, chain scission takes place. The physical properties of the polymer deteriorate and its average molecular weight (chain length) decreases, melt flow rate increases and a powdery surface eventually forms. Polymer degradation is a natural phenomenon that cannot be totally stopped. Instead, resin producers seek to stabilize the colour and physical properties of their polymers for a reasonable life span, which varies depending on the end user requirements. Auto-oxidation continues unless countermeasures are taken to halt the process. One way to terminate auto-oxidation is by adding various antioxidants to the resin. Antioxidants (A/O) are a class of chemicals with varying chemical compositions and methods of terminating auto-oxidation. The antioxidant chemicals are additives for specific operation; other additives are widely used for other polymer properties modifications. Additives such as heat stabilizers, light stabilizers, slip agents, plasticizers, and antioxidants represent some of the most common classes of compounds used to tailor polymer properties for specific applications. So, commercial final polymer products should contain some types of additives, these chemical additives may be reacting with some or all environmental elements. For example; acid rains may react with the antioxidant at the surface (etches the surface), and hence the surface will be opened to the UV radiation attack, and so on. Therefore, studying the effect of UV radiation on polymer samples after wetting them in acid rain simulating solution is very important step for evaluation the efficiency of the used antioxidant. With the same logic, in order to examine the chemical resistance of a polymer against acid rain, the polymer sample should be exposed to UV radiation before the examination. 


\section{EXPERIMENTAL MEASURES:}

The polymer used in the current study is commercial polyethylene films that are manufactured in Egypt for plants greenhouses usage. Plasticizer, antioxidant, UV stabilizer, antistatic charging and slip agents are added to the used $\mathrm{PE}$ films with unknown ratios. Film thickness is $0.08 \mathrm{~mm}$

\section{1- Sample treatments:}

The commercial PE film was cut into small pieces each of area equivalent to that of the A4 paper sheet. These pieces are divided into four groups plus one piece that is taken as blank sample (denoted later as fresh film) which is not treated at all. Each group is subjected to a specific treatment as following:

First group: consists of 6 pieces, all pieces are immersed in big basin filled with acid rain simulating solution. After one week one piece is picked out the solution and washed with the tap water and then left to dry in oven at about $40 \mathrm{oC}$ for 2 hours. After two weeks the second piece is drawn from the basin and dried too, and so on for the rest of group samples. Samples of this group are denoted as (AR 1-6). Exposure time in acid rain is considered in units of week.

- Second group: consists of 6 pieces, one piece is irradiated by intensive UV radiation for one day and then drawn out of radiation stream. The next piece is irradiated for two days, and so on for the rest of this group samples. Samples of this group are denoted as (UV 1-6). Exposure time in UV radiation is considered in units of day.

- $\quad$ Third group: consists of 6 pieces, one piece is immersed in the acid rain solution for one week and dried, then it is irradiated by same UV for one day. Second piece is immersed in the acid rain solution for two weeks and then irradiated by the UV radiation for two days, and so on for the reset pieces of this group. Samples of this group are denoted as (AR \& UV 1-6).

- $\quad$ Forth group: consists of 6 pieces, one is irradiated by the same UV for one day and then immersed in the acid rain solution for one week and dried. Second piece is irradiated by the UV radiation for two days, and then immersed in the acid rain solution for two weeks then dried, and so on for other pieces of the group. Samples of this group are denoted as (UV\&AR 1-6).

\section{2 - The acid rain simulator}

(Schulz, et al, 2000) showed that, the pHs of a real acid rain even at the aggressive environments lied in the range of 3.5-4.5. Acid rain etches the acrylic polymer and strongly damages the surface coating [11]. Another authors said that acid rain is formed when the $\mathrm{pH}$ of rain water is below 5.6. As the literature does not provide any exact specification for the

www.ijsea.com chemical content of the acid rain, it is usually assumed to be the mixture of sulphuric acid and nitric acid of $\mathrm{pH} 4.3-4.5$ [12]. Therefore a mixture of sulphuric and nitric acids of $\mathrm{pH} 3.6$ was prepared and used in this work as acid rain simulator to represent the aggressive acid rain that is normally characterizing industrial zones and around classical power stations. The $\mathrm{pH}$ values were measured by a digital $\mathrm{pH}$-meter model 350 from Jenway Co, with glass probe.

\section{3 - UV source}

The used UV radiation source containing three germicidal UV lamps from General Electric Co., model G15T8, $436 \mathrm{~mm}$ length, $25.5 \mathrm{~mm}$ diameter, $15 \mathrm{~W}$ radiation powers and $254 \mathrm{~nm}$ characteristic wavelength for 10,000 average useful life. The distance between the light source lamps and samples is $(15 \mathrm{~cm})$. Each sample is exposed to this radiation for different periods ranged from 1 to 6 days, continuously.

\section{4 - Infrared spectrophotometer}

Infrared spectroscopy is a versatile experimental technique and it is relatively easy to obtain spectra from samples in solution or in the liquid, solid or gaseous states. The first dispersive infrared instruments employed prisms made of non-absorbing materials for IR-wavelengths such as sodium chloride. The popularity of prism instruments fell away in the 1960s when the improved technology of grating construction enabled cheap, good quality gratings to be manufactured [13]. In the present work, an instrument model NICOLET- IS10 from Thermo Scientific Co., USA is used. Polymer samples from each sample group were examined after exposure to the selected treatment for detecting the molecular changes, if any.

\section{5 - X - Ray diffraction measurements}

$\mathrm{X}$ - ray diffraction is a powerful tool for the identification of the crystallization state of solids. The crystallization state includes degree of crystallization, crystal size, crystals type, rate of crystallization and crystal distribution as special case. The X-ray diffraction charts may provide well information about the macro-molecular orientations. The mean orientation factors (k) may be calculated as the ratio between intensity of crystalline maxima for samples treated and untreated analogues [14]. The x-ray tube that used in the present work was adjusted at the following measurement conditions: $\mathrm{Cu}$ target, voltage of $40 \mathrm{kV}$ at $30 \mathrm{~mA}$. (Shimadzu6000, Japan).

\section{6 - Thermal analysis}

Differential Scanning Calorimetric (DSC) technique is widely used to investigate the properties of polymers upon heating on certain range at specific rate. Melting and crystallization are examples of phase transitions of the first order. This means a transition where the heat capacity as a function of time and temperature exhibits a discontinuity, which has the shape of a peak. The area under the peak in the thermo-gram is directly proportional to the energy that is emitted or absorbed during changes of state in the sample. The highest point in the peak reflects the temperature or time when 
the largest part of a sample is melting or crystallizing or when a polymerization or curing reaction occurs at its highest rate. Differential thermal analysis (DTA) is also used when the melting point is the task. DTA is excellent technique for the determination of melting temperature of crystalline polymers, where the sharpness of melting peak can represent the degree of crystallinity. Therefore this technique is in-efficient in the case of amorphous (glassy) polymers.

In the present work, the Shimadzu50 (Japan), instrument was used in atmosphere of nitrogen and gas flow of $20 \mathrm{ml} / \mathrm{min}$ for sample of weight $10 \mathrm{mg}$ in aluminium pan.

\section{7 - Mechanical Stress - Strain Characterization}

There are three factors determine whether a polymer is glassy, rubbery or fibre-forming under a given set of conditions. These are the chain flexibility, the chain interattraction and the regularity of the polymer. The relationship has been expressed diagrammatically by Swallow [15]. Failure of polymers in certain applications to carry design loads or occasional accidental overloads may be due to excessive plastic deformation resulting from the inadequate strength properties of the polymer. For the quantification of such failures, the mechanical property of primary interest is the yield strength and the corresponding strain. The ultimate strength, along with the associated strain, also provides useful information [16].

The machine used to measure the recent mechanical properties is Zwick Tensometer Z010, Germany, with calibrated load cell at temperature of $23+0.20 \mathrm{C}$ and R.H. of $45+5 \%$.

\section{RESULTS AND DISCUSSION}

When a material (say polymer) is attacked by acid, the first expectation is occurrence of a chemical reaction. The easier technique to investigate any chemical changes in a material is the IR-spectroscopic analysis. Therefore, PE samples that are subjected to the acid rain (AR) were tested; figure 3 shows the transmittance percent of the AR-group samples.

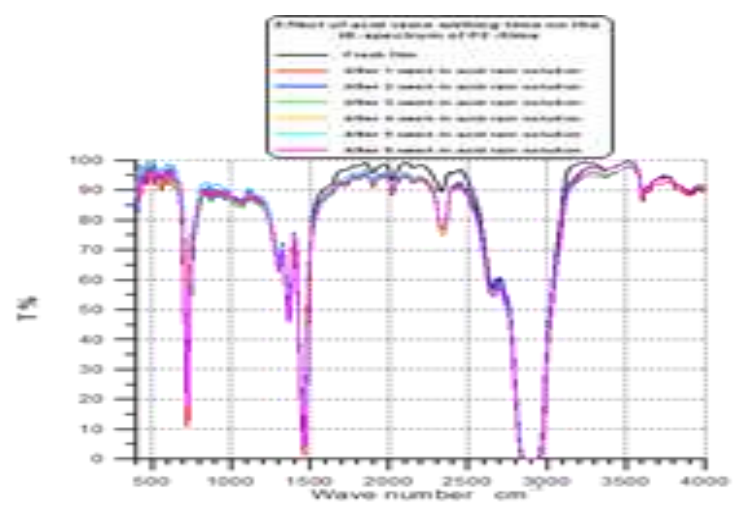

Figure (3): IR - Transmission spectrum of PE samples subjected to acid rainsolution for different periods ranged from 1 to 6 weeks.
Through the first look it is clear that no serious chemical changes have been occurred, which is expected due to the high chemical resistance of PE. A careful evaluation of the spectrum one deduces the etching effect of the acid rain where the PE sample surface became smoother and may be thinner. This is clear from the increasing in contrast of transmitted interference fringes due multi-reflections between the parallel surfaces of the sample as shown in figure 4 . The greatest etching effect takes place during the first week, which means that the functional additives used in this commercial PE are acid reactive.

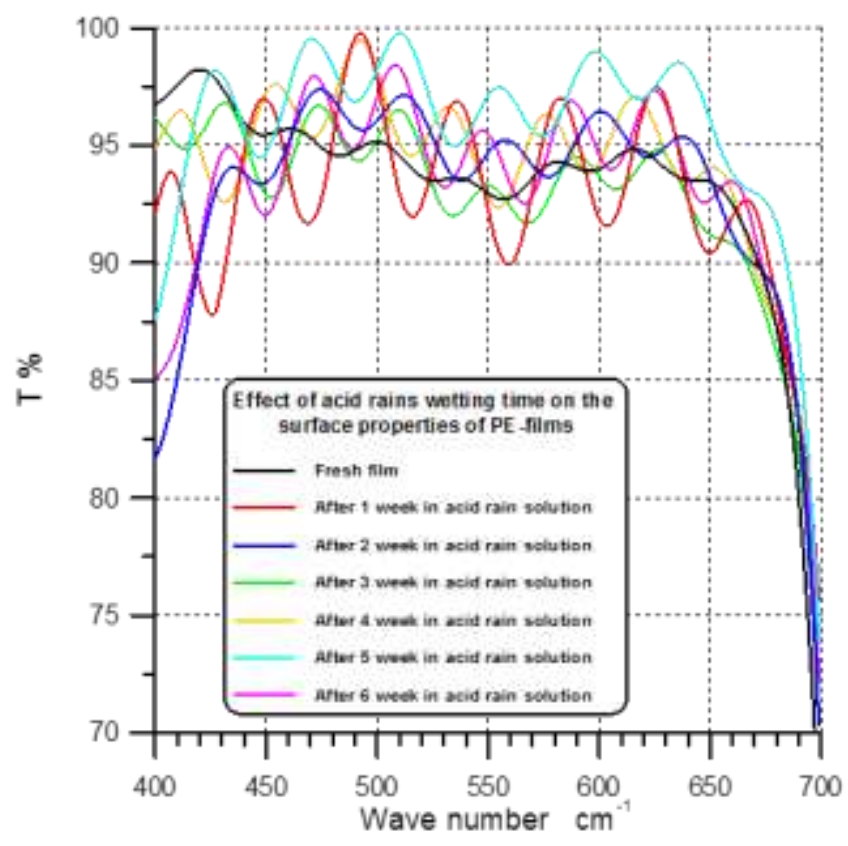

Figure (4): The etching effect of acid rain on the surface optical propertiesof commercial PE samples.

Also, due to the known oxidative degradation of polymers by UV - irradiation, it is expected to observe some oxygen bonds in the structure of PE. The IR-spectrum of UV irradiated PE shows that bond at $1720 \mathrm{~cm}-1$, as shown in figure 5 . 


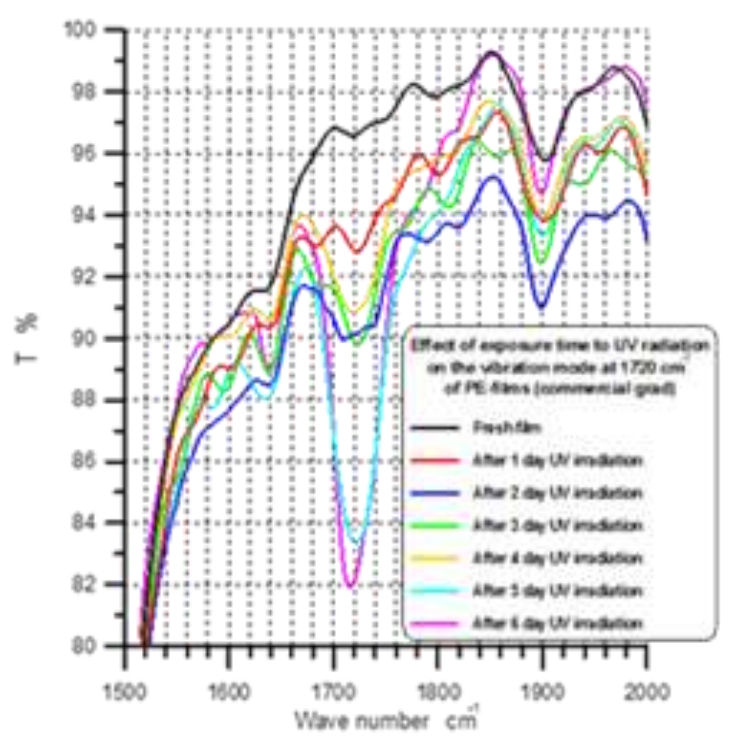

Figure (5): The appearance of $\mathrm{C}=\mathrm{O}$ bond in the UV-irradiated $\mathrm{PE}$ structureafter 3 days of continuous irradiation.

From figure 5 it is clear that the added UV stabilizer is of medium efficiency since it could withstand the intense UV radiation along three days, but after 5 days the efficiency of this stabilizer failed. Figure 6 shows that the added UV stabilizer in the used commercial PE samples was among the chemicals that are removed during the etching effect of the acid rain. The red curve on figure 6 represents the strong existence of the oxide bond ( $23 \%$ on the transmittance scale) by the UV irradiation after the acid rain etching, while the blue curve represents the weak oxidation bond ( $8 \%$ on the transmittance scale) by UV radiation before the etching by acid rain.

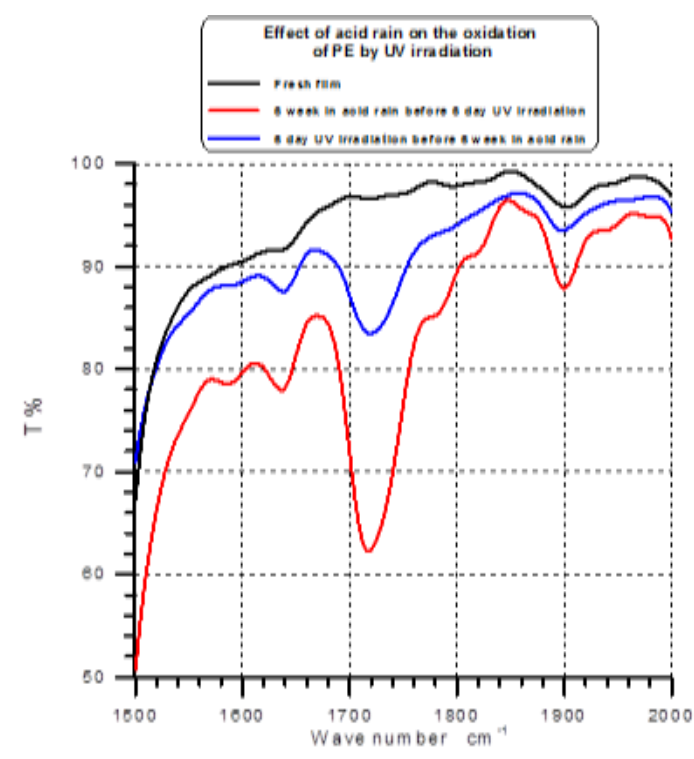

Figure (6): The effect of acid rain etching on the oxidation of PEby UV radiation.
The behaviour shown in figure 6 illustrates that acid rains have reacted and removed the UV stabilizer, then the polymer molecular system became unprotected against UV radiation, and hence when UV radiation attacks the polymer after the acid rains it could to degrade the polymer seriously.

That previous discussion shows the great advantage of studying the effect of successive exposure to environmental elements over the individual studies of each element.

Sequenced effects of environmental elements on polymer materials are not enclosed in the polymer degradation by chemical reactions but these effects are extended to the morphological structure of the polymer molecular system too.

$\mathrm{X}$-ray diffraction technique is among the best methods of evaluating morphology of solid objects. Figure 7 represents the effect of acid rain and UV radiation on the used commercial PE films, individually. The graph shows that exposing PE films to acid rain for 6 weeks induced crystalline enhancement equivalent to that induced by UV irradiation for 6 days. There is no new crystalline structures are observed.

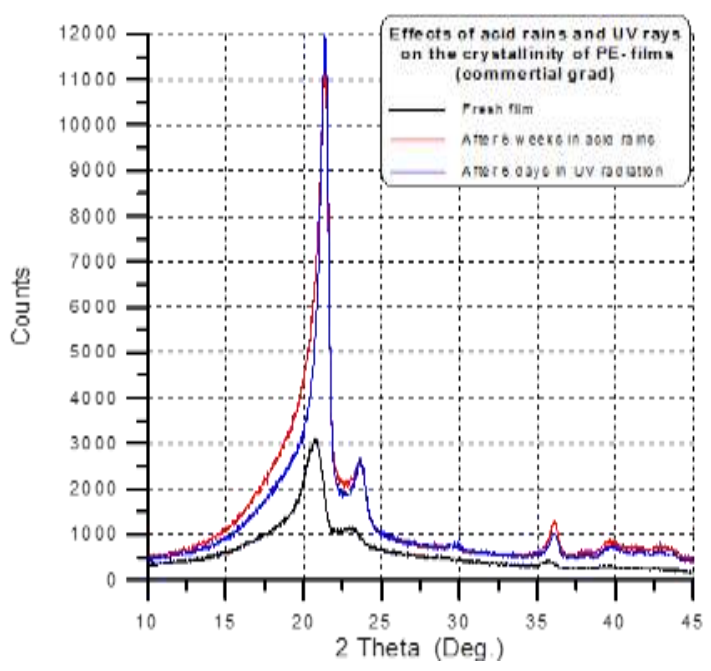

Figure (7): The maximum changes in crystalline state of PE due to acid rain and UV radiation individually.

Figure 8 shows the etching effect of the acid rains on the morphology of PE films; where after etching, the UV could to penetrate the sample with higher intensity and causes more crystalline enhancement. 


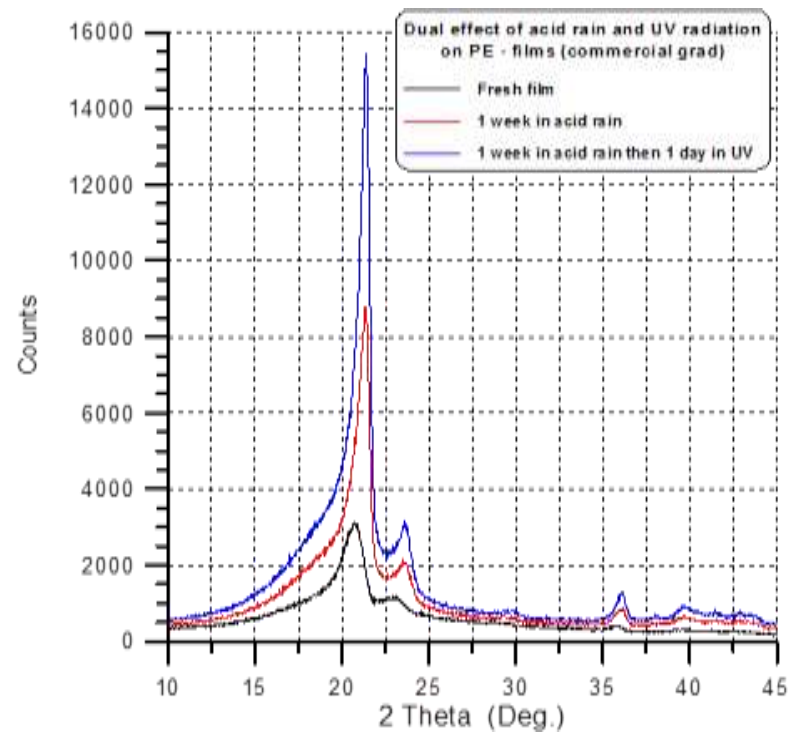

Figure (8): The effect of the etching by the acid rain on the crystalline state of PE films.

From figure 8, it is noted that one week for the sample in acid rain caused limited enhancement in the crystalline state of the polymer sample, but if this sample is subjected to UV radiation after acid rain, the crystalline state of sample material possesses a great enhancement. Figure 9 shows the same effects as discussed above but for reversed sequence.

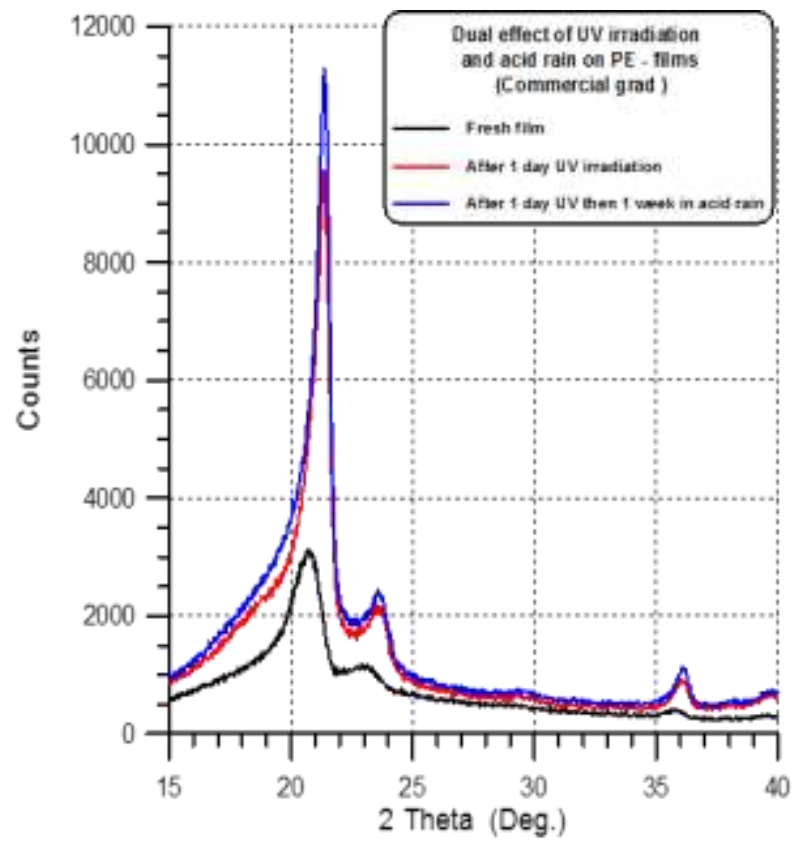

Figure (9): Successive effect of UV radiation and acid rain on the crystalline state of PE films.
It is clear from figure 9 that when acid rain attack follows the UV attack, its effect on the crystalline state of PE sample is very limited. Comparing graphs in figures 8 and 9 with graphs in figure 7 , one deduces that the most of morphological variations by acid rain and UV radiation are tack place within the first day of UV and the first week of acid rain exposure times.

It is well known that any variations in the morphological state of a material should show reflections on the thermal properties as well as the mechanical properties of that material. For a single material, there are several thermal properties. The DTA thermo-gram provides information about two different parameters simultaneously, namely; melting point (at the sharp tip of the melting peak) and the relative amount of the present crystalline phase (as the length of the melting peak).

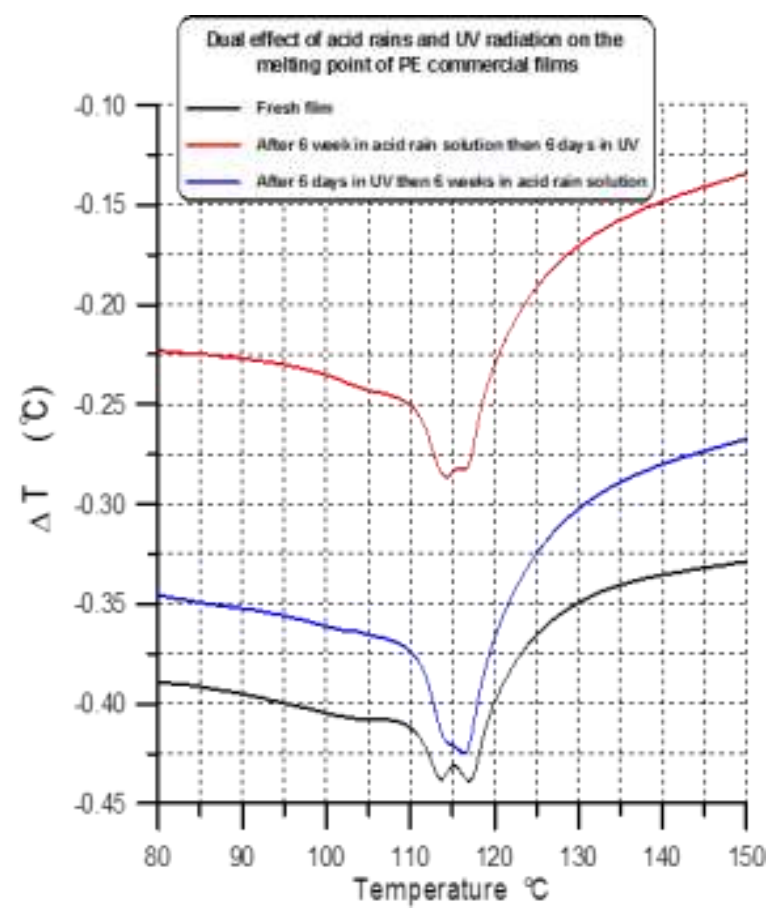

Figure (10): DTA thermo-gram of PE film sample exposed to acid rain and UV radiation in different sequences.

Figure 10 shows that the melting point of PE is $115 \mathrm{oC}$ and neither the acid rain nor the UV irradiation has changed this value. This result is confirmed from figures 7 to 9 , where the crystals type not changed and no new crystalline phase has been formed due to the external effects. It is noted from figure 10 also that the melting peak of the samples, which exposed to acid rain and UV radiation, are somewhat longer due to the increase of the crystalline amount in the sample by the UV radiation and acid rain. The selected exposure time for both acid rain and UV radiation show limited observable changes in the length of melting peaks (as shown in figure 11), which in contrast with the observable peak changes in the X-ray diffraction patterns. 


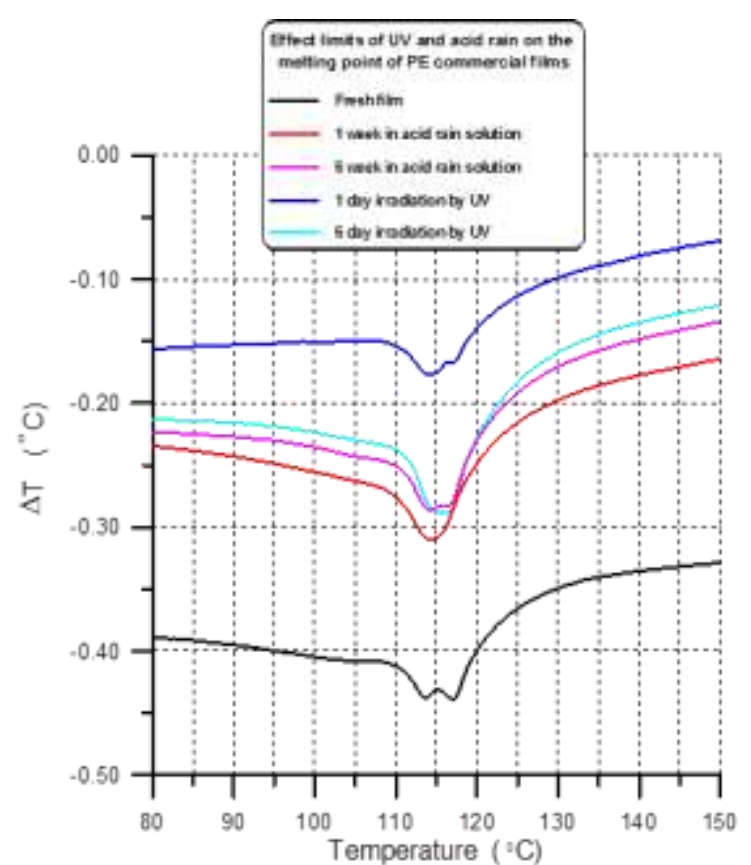

Figure (11): Limited changes in the length of melting peaks through the range of exposure time to acid rain and UV radiation.

Mechanical properties of the PE samples show variations due to the exposure to acid rain and UV radiation, too. Tables (13 ) concluded the observed changes during the tensile test of all samples.

Table (1): The percentage elongation at the yield point

\begin{tabular}{|c|c|c|c|c|}
\hline $\begin{array}{c}\text { Time } \\
\text { interval }\end{array}$ & UV & AR & $\begin{array}{l}\text { AR then } \\
\text { UV }\end{array}$ & $\begin{array}{c}\text { UV then } \\
\text { AR }\end{array}$ \\
\hline 0 & 12 & 12 & 12 & 12 \\
\hline 1 & 9.2 & 12 & 7.3 & 10.9 \\
\hline 2 & 10.7 & 9.2 & 5.7 & 9.0 \\
\hline 3 & 5.7 & 8.9 & 7.0 & 6.6 \\
\hline 4 & 5.7 & 8.75 & 3.3 & 4.6 \\
\hline 5 & 6.6 & 8.8 & 3.3 & 3.9 \\
\hline 6 & 3.6 & 9.4 & 3.9 & 3.3 \\
\hline \multicolumn{5}{|c|}{$\begin{array}{l}\text { NOTES: } 1-\mathrm{AR}=\text { exposure to acid rain, } 2 \text { - UV = } \\
\text { exposure to ultra violet radiation } \\
\text { 3- time intervals are in units of weeks for AR and in days } \\
\text { for UV }\end{array}$} \\
\hline
\end{tabular}

Table (2): The percentage elongation at the breaking

\begin{tabular}{|c|c|c|c|c|}
\hline $\begin{array}{c}\text { Time } \\
\text { interval }\end{array}$ & UV & AR & $\begin{array}{c}\text { AR } \\
\text { then } \\
\text { UV }\end{array}$ & $\begin{array}{c}\text { UV } \\
\text { then } \\
\text { AR }\end{array}$ \\
\hline 0 & 960 & 960 & 960 & 960 \\
\hline 1 & 980 & 960 & 870 & 825 \\
\hline 2 & 780 & 840 & 380 & 625 \\
\hline 3 & 630 & 880 & 290 & 520 \\
\hline 4 & 590 & 780 & 75 & 220 \\
\hline 5 & 135 & 780 & 75 & 123 \\
\hline 6 & 75 & 890 & 74 & 80 \\
\hline NOTES: 1- AR = exposure to acid rain, \\
\hline 2- UV = exposure to ultra violet radiation \\
3- time intervals are in units of \\
weeks for AR and in days for UV \\
\hline
\end{tabular}

Of course, all of the observed variations in the mechanical parameters of $\mathrm{PE}$ films should be related to formation of the strong $\mathrm{C}=\mathrm{O}$ as well as the dramatic increase for the amount of crystalline phase by exposure to acid rain followed by UV irradiation. The increase in the crystalline phase makes the material more brittle and hence less ductility, which is clear from the continuous decrease in all tensile parameters. The effect of etching by acid rain appears in the fast and great downfall of the percentage elongation at breaking of the samples (AR then UV) in table (2). This set of tables shows that acid rain alone did not affect the mechanical properties of PE seriously, which supports the high chemical resistance of the PE. These tables show also that the used UV-stabilizer in the commercial PE samples under tests is of medium efficiency, where sample`s mechanical properties resisted UV radiation for maximum 3 days and then downfall.

As mentioned above, the observed variations in the mechanical parameters are symptoms of two factors, which are the formation of $\mathrm{C}=\mathrm{O}$ bonds (or oxidation) and the increase of crystalline phase. The question arises here is, how one can identify the share or responsibility of each factor in the observed variations? The answer may be found in the rate of changes. As seen in figure 5, the oxidation of PE increases gradually as time increases, this behaviour is coincident with that shown in table 2 for the UV coulomb. Thus one may consider that the variation of percentage elongation at break belongs to the oxidation progress in PE. On the other side, figure 8 shows that most of the crystalline phase enhancements are occurred during the beginning of the action periods, which is coincident with data changes in table 1 for (AR then UV) coulomb, where data changed suddenly after the first time step. Thus one may consider that the variation of percentage elongation at the yield point belongs to the change in size of the crystalline phase.

Generally, in the current case, the rate of oxidative degradation (by UV radiation) is proportional to the rate of changing the 
percentage elongation at breaking and the rate of crystalline phase enhancement is proportional to the rate of changing the percentage elongation at the yield point through the tensile test.

\section{CONCLUSION}

Utilization of polymer products for outdoor applications is continuously increasing. So the stability of polymers against environmental degradation became top of interests for many researchers. The acid rains, UV radiation and heat are the most degrading environmental elements. The effect of these elements on the polymers stability has been studied, but individually. The authors of the current work clam that solutions derived from these individual studies are of limited efficiency, because of their conflicts. The current study clarifies a type of these conflicts. The traditional solution for the UV degradation of polymers is to add certain organic chemical additive to increase the polymer resistance for the UV degradation. Acid rains may react with these chemical additives and restricts their activity against UV radiation, and hence the polymer article becomes unprotected against UV. This work shows that successive exposure to acid rain and UV radiation can minimize the efficiency of some chemical additives. It is better to study effects of all environmental elements in sequenced manner and all collected data should be analysed at once. By this suggested researching regime, a unique high efficient solution can be established. It is found that acid rains have etching effect on the polymer surfaces. Acid rains alone caused no serious degradation, which is due to the high chemical resistance of the used PE polymer. Other polymers, say glassy polymers, may not resist the acid rains attack. Major effects of acid rains are occurred within the first week, longer exposure time to acid rain causes minor effects. In contrast, degradation effects by UV radiation are extended as long as the radiation attacks the polymer until full degradation of the polymer. So, it is recommended, after this study, to prevent accumulation of acid rains on the polymer films for long time.

\section{REFERENCES}

[1] - Rabek J.F., Comprehensive Chemical Kinetics, Vol. 1, Degradation of Polymers, Ed. C. H. Bamford, C. H. F. Tipper, [Elsevier Scientific Publishing Company, Amsterdam] (1975)

[2] - Bamford C.H., Tipper C.H.F. (eds.), Comprehensive Chemical Kinetics, Vol. 14, Degradation of Polymers, [Elsevier Scientific Publishing Company, Amsterdam] (1975)

[3] - Jellinek H.H.G. (ed.), Aspects of Degradation and Stabilization of Polymers, [Elsevier Scientific Publishing Company, Amsterdam] (1978).

[4] - Allen N.S. (ed.), "Degradation and Stabilization of Polyolefins", [Applied Science Publishers Ltd., London and New York] (1983)

[5]- Scott G. (ed.) "Atmospheric Oxidation and Antioxidants" Vol. I, [Elsevier Publishing Company, Amsterdam] (1993)
[6] - Schnabel W., Polymer Degradation; Principles and Applications, [Macmillan, New York] (1981)

[7]- Reich L., Stivala S.S., Elements of Polymer Degradation, McGraw-Hill Book Company, (1971)

[8] - Peacock A. J., "The chemistry of polyethylene", Journal of Macromolecular Science, Part C: Polymer Reviews, vol. 41(4), pp. 285-323 (2001).

[9] - Feldman D., "Polymer weathering: Photo-oxidation", Journal of Polymers and the Environment, vol. 10, pp. 163-173 (2002).

[10] - Ranby B. and Rabek J. F., "Photodegradation, photooxidation and photo- stabilization of polymers", [John Wiley \& Sons, London, UK] (1975).

[11] - Schulz, U.; Trubiroha, P.; Schernau, U.; Baumgart, H., J.Progress in Organic Coatings, vol. 40, pp. 151-165, (2000)

[12] - CHEMIK, vol. 66, 4, 315-320, (2012).

[13] - Stuart B., "Infrared Spectroscopy: Fundamentals and Applications", (John Wiley \& Sons, Ltd, New York, London, ISBN: 0-470-85427-8) (2004).

[14] - António R., Guerreiro, Vadim Korkhov, Irene Mijangos, V. Elena, Piletska, Juris Rodins , Anthony Turner, P. F. and Sergey A. Piletsky., J. Biosensors

and Bioelectronics, vol. 23. Issue 7, pp. $1189-1194$, ISSN 0956-5663,(2008).

[15] - SWALLOW C., J. Roy. Soc. Arts, vol. 99, pp. 355 (1951).

[16] - Robert O. Ebewele, "Polymer Science and Technology ", (CRC Press LLC, Boca Raton and New York) Chapter 13, (2000). 